\title{
Acute Intradialytic Complications in End Stage Renal Disease on Maintenance Hemodialysis
}

\author{
Agrawal RK, ${ }^{1}$ Khakurel S, ${ }^{1}$ Hada R, ${ }^{1}$ Shrestha D, ${ }^{1}$ Baral A' \\ 'Department of Nephrology, National Academy of Medical Sciences, Bir Hospital, Kathmandu, Nepal.
}

\section{ABSTRACT}

Introduction: Hemodialysis is the preferred method of treatment for Nepalese patients with End Stage Renal Disease. Despite the technological advances and better understanding of physiology associated with hemodialysis, a number of complications are known to be associated with hemodialysis. This study was undertaken to study the frequency of acute intradialytic complications in patients undergoing hemodialysis.

Methods: A cross-sectional study was conducted at Nephrology unit of a tertiary care hospital from 15 June, 2007 to 15 December, 2007. A total of 28 patients were included in the study. Patients with acute renal failure and acute on chronic renal failure were excluded from the study.

Results: Total sessions of hemodialysis during the period were 1455 . Hypotensive episode were $66(4.5 \%)$ and was the commonest complication and followed by hypertensive episodes were $58(3.8 \%)$. Other problems encountered were transfusion reactions were 23 episodes $(1.5 \%)$, rigors not related to transfusions were 13 episodes $(0.8 \%)$, nausea/vomiting were 20 episodes $(1.4 \%)$, muscle cramps were 12 episodes $(0.8 \%)$, hypoglycemia were 6 episodes $5(0.4 \%)$.

Conclusions: The frequency of intradialytic complications is low and many of them are not life threatening.

Keywords: complications; end stage renal disease; hemodialysis; hypertension; hypotension.

\section{INTRODUCTION}

Chronic Kidney Disease (CKD) is defined as kidney abnormalities that persist for at least three months, with or without decreased GFR. The commonest manifestation of kidney damage is persistent albuminuria. ${ }^{1}$ Symptoms of uremia occur when GFR is below $15 \mathrm{ml} / \mathrm{min} .^{2}$ CKD is a common and rapidly increasing public health problem all over the world including Nepal. Patients with end stage renal disease (ESRD) require renal replacement therapy for survival. Hemodialysis for RRT was started in the early 1960 s by Scribner. ${ }^{3}$ In Nepal, hemodialysis was started in $29^{\text {th }}$ September 1988 at Bir Hospital. ${ }^{4}$
The common complications during hemodialysis include hypotension, cramp, nausea and vomiting, headache, chest pain, back ache, pruritus, fever and chills. ${ }^{5}$ The aim of this study was to find out the frequency of these complications at Bir Hospital.

\section{METHODS}

A cross-sectional study was conducted at the Department of Nephrology, Bir Hospital from 15 June to 15 December 2007. All patients with diagnosis of

Correspondence: Dr. Rajendra Kumar Agrawal, Department of Nephrology, NAMS, Bir Hospital, PO Box No 21821, Kathmandu, Nepal. Email: rajendraanju27@gmail.com, Phone: 9851023840. 
ESRD and undergoing maintenance hemodialysis were included in the study. Patients with ARF and acute on chronic renal failure were excluded from the study. A total of 28 patients were followed up for the study period and any complications during dialysis were recorded. Patient's symptoms, nurses records and physicians interventions were analyzed to sort out the possible complications. Hypotension was defined as any recorded blood pressure of less than $20 \mathrm{~mm}$ of $\mathrm{Hg}$ fall in systolic BP from the baseline blood pressure during the dialysis session, $90 / 60 \mathrm{~mm}$ of $\mathrm{Hg}$ with or with-out symptoms. Intradialytic hypertension can be defined as an average pre to post hemodialysis systolic blood pressure elevation of $>10 \mathrm{mmHg}$ for more than $4 / 6$ of the last dialysis treatment sessions, BP of more than $160 / 100$ irrespective of the number of antihypertensive medications. The frequency of cramps, rigors, transfusion related reactions, hypoglycemic episodes were recorded. In addition to the above mentioned acute complications some common chronic complications like ascites, pruritus, recurrent infection and anemia assessed by the number of transfusions were also noted during the study period.

To assess the adequacy of Hemodialysis $\mathrm{Kt} / \mathrm{v}$ of individuals were calculated by the plasma urea reduction ratio using the formula of Jindal and Manuel, ${ }^{6}(\mathrm{Kt} / \mathrm{v}=\mathrm{PRU} \times 0.04-1.2)$ where PRU means ( PRU = Pre-dialysis Blood urea- Post dialysis Blood urea/ predialysis Blood urea $x$ 100). Data were entered in to an excel worksheet and analyzed. Ethical approval was taken.

\section{RESULTS}

A total of 28 patients were included in the study. The mean age of the patients was 48.78 years. The minimum age was 25 years and maximum was 71 years. Male patients were predominant in the study which included $68 \%$ of the total patients and females accounted for only $32 \%$. Hypertensive nephrosclerosis was the commonest condition leading to ESRD in 12 (39\%) patients. This was followed by Chronic Glomerulonephritis in 9 (32\%) and diabetic nephropathy in $5(21 \%)$ patients. Autosomal Dominant Polycystic Kidney Disease (ADPKD) in $1(4 \%)$ and obstructive uropathy in 1 (4\%) accounted for the rest. Majority of the patients were receiving anti-hypertensive medicines in various combinations. 10 out of the 28 patients received 3 drug combinations and 8 patients needed 4 drugs to control their blood pressure. Similarly 5 patients required 2 drug combination and 3 patients had just 1 and 1 patient require 5 drugs for controlling the blood pressure. The median of interdialytic weight gain was 8-9 pounds. Most patients received 2 sessions of hemodialysis per week each session lasting for 4 hours.
However 5 of the 28 patients required emergency dialysis in between, amounting to emergency dialysis of 10 sessions during the study period. All patients received bicarbonate based dialysis. Six patients required hospital admissions for various complications and there were a total of 12 admissions during the study period. Mortality among the study patients was quite high $(57.1 \%)$ (Table1). The causes of death were septicemia in 5 (31.25\%), hyperkalemia in 5 (31.25\%), acute pulmonary edema in $4(25 \%)$ and disseminated tuberculosis in $2(12.75 \%)$ patients.

Table 1. Table showing the causes of death in the study group.

\begin{tabular}{ll}
\hline Cause of death & No. $(\%)$ \\
Septicemia & $5(31.25 \%)$ \\
Hyperkalemia & $5(31.25 \%)$ \\
Pulmonary edema & $4(25 \%)$ \\
Disseminated tuberculosis & $2(12.5 \%)$ \\
\hline
\end{tabular}

Total hemodialysis sessions were 1455 during the six months of the study. Among the intradialytic complications 66 hypotensive episodes (4.5\%) was the commonest. This was followed by 58 episodes of hypertension (4\%), 12 episodes of muscle cramps $(0.8 \%), 23$ episodes of transfusion reactions $(1.5 \%)$, 20 episodes of nausea/ vomiting (1.4\%), and 13 episodes of chills and rigors not related to transfusions (0.8\%) (Table 2).

Table 2. Table showing the important intradialytic complications and their frequency.

\begin{tabular}{ll}
\hline Event & Frequency (\%) \\
\hline Total Hemodialysis sessions & 1455 \\
Hypotensive episodes & $66(4.5)$ \\
Hypertension & $58(3.9)$ \\
Transfusion reaction & $23(1.5)$ \\
Nausea/ vomiting & $20(1.4)$ \\
Cramps & $12(0.8)$ \\
Rigors & $13(0.8)$ \\
Hypoglycemia & $6(0.4)$ \\
\hline
\end{tabular}

Severe cardiac arrhythmias, symptomatic hypoxia and minor complications like chest pain associated with hypotensive episodes and backache were not reported during the hypertension study. Hypoglycemia was seen in two patients and one patient suffered 5 episodes of hypoglycemia despite being non diabetic. Only one patient with diabetic nephropathy was continuing on insulin therapy for control of blood sugar levels.

Infection and anemia were the major interdialytic complications. Pneumonia was seen in $80 \%$ of total 
infections and Urinary tract infections accounted for the rest. Majority of patients was dependent on blood transfusions for management of anemia and there were a total of 228 units of blood transfusion. On average a patient was transfused 12 times during the six months ( 2 units of blood per month). Pruritus was seen in $25 \%$ of patients while malnutrition was diagnosed in $22 \%$ of patients. One patient underwent coronary artery bypass surgery for coronary artery disease while one patient developed thrombocytopenia and one had Anti $\mathrm{HCV}$ positive status. Idiopathic ascites was seen in 15 (54\%) out of 28 patients.

To assess the adequacy of dialysis $\mathrm{Kt} / \mathrm{v}$ was calculated for each patient and majority had inadequate dialysis characterized by a $\mathrm{Kt} / \mathrm{v}$ of less than $1.2(75 \%)$ (Figure 2).
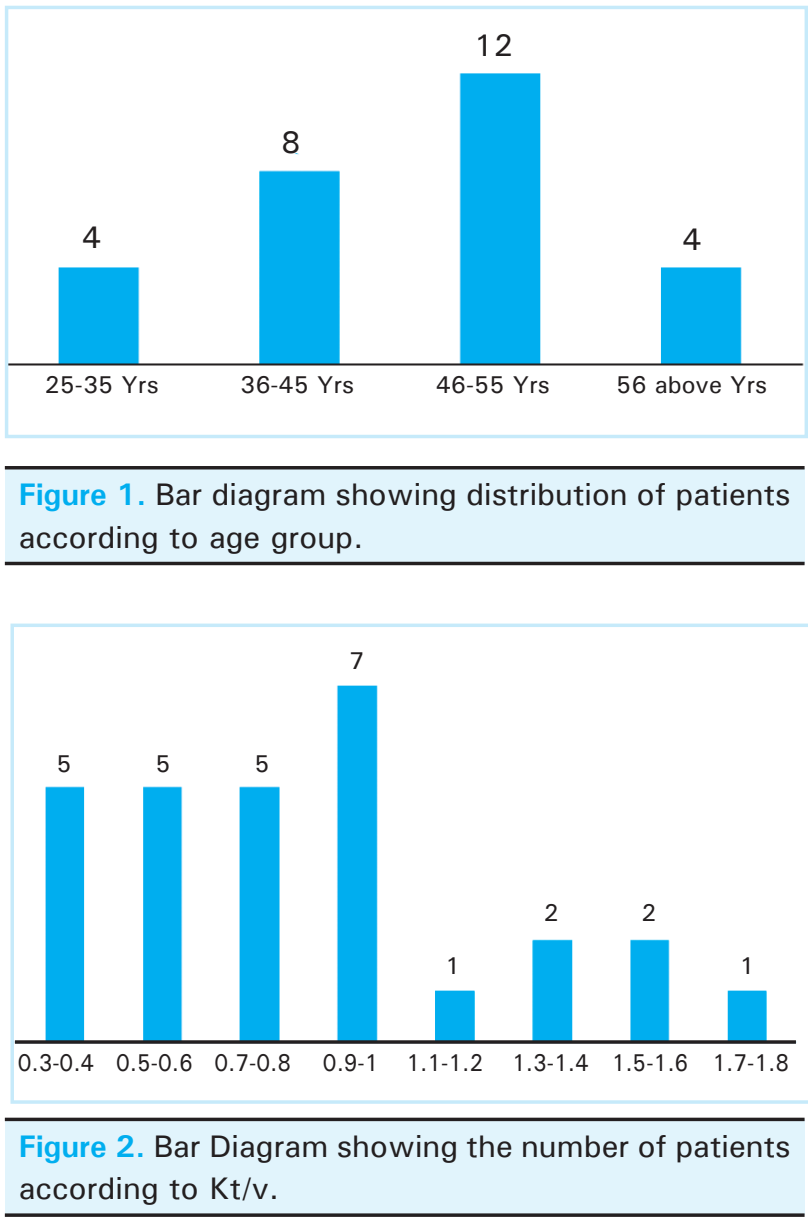

\section{DISCUSSION}

Hypotension which is the commonest complication during hemodialysis is due to many factors like the size of extracorporeal circulation, degree of ultrafiltration, changes in serum osmolality, autonomic neuropathy and alteration in body temperature due to dialysis temperature. ${ }^{7}$ It's incidence has been reported from less than $5 \%$ to $40 \%$ of all treatments. In our study this was $4.5 \%$ of total hemodialysis sessions. It affected around $45 \%$ of patients undergoing hemodialysis at some time or other. Hypotension alone consisted about $33 \%$ of total complications. This is lower than the value reported at Nepal medical college teaching hospital in Kathmandu. ${ }^{8}$ The population characteristics are very similar to ours but the difference in reporting may be due to variation in definition which can vary from symptomatic hypotension requiring active intervention to asymptomatic percentage in fall of blood pressure. Furthermore they have not mentioned about the frequency of hypotension in relation to total treatment sessions. This finding also does not correlate with the findings by of Pakistan where they reported the incidence of hypotension to be as high as $54.6 \%$ in females and $45.5 \%$ in males. ${ }^{9}$ No sex wise difference was noted on the incidence of hypotension in the present study.

Hypertension which occurs usually towards the end of hemodialysis treatment was another frequent complication seen in our patients. Hypertension occurs due to the activation of Renin Angiotensin Aldosterone Axis. It is known to affect up to $15 \%$ of hemodialysis patients and frequently seen in older patients with lower dry weighs and those on more antihypertensive medications and those with lower serum creatinine levels. ${ }^{10}$ In our study this complication was seen in $3.8 \%$ of total treatments. It was seen in $50 \%$ of patients and included $25 \%$ of all complications which is similar to the value reported by in Nepal Medical College study. ${ }^{8}$ The reason for lower incidence of hypertension in our patients may be due to younger age group and higher serum creatinine values as dialysis induced hypertension is seen commonly in older patients and those with lower serum creatinine values.

Blood transfusion was the major modality of management of anemia in our patients and transfusion related problems were quite common in this group. The reactions were not graded in terms of severity but $1.5 \%$ of all transfusions was complicated by reactions and required intervention of some sort.

Febrile reactions including rigors, chills are also known complications of hemodialysis independent of the transfusion status of the patients. These reactions are believed to occur due to activation of leukocytes, macrophages, platelets, complement cascade and the coagulation cascade as the blood moves through the extracorporeal system. The incidence of these reactions is also reported to be as high as $45 \% .{ }^{10}$ As with other complications this was also much lower in our patients and accounted for only $0.8 \%$ of the total treatments. Muscle cramps were another frequent complication 
noted in our patients. It is frequently related to acute contraction in intravascular volume and is known to affect around $20 \%$ of dialysis treatments. ${ }^{11}$ The frequency of this complication was only $0.8 \%$ in our patients.

Hypoglycemia either as a complication of ant diabetic medications or spontaneous due to poor nutritional status is another known complication of hemodialysis. This was seen in $0.4 \%$ of total treatments. In fact 5 of the 6 reported episodes were in one single patient who was not a diabetic.GIT complications like nausea and vomiting are also commonly seen during hemodialysis. Most of the times they arise as symptoms of hypotension. In the study by David and Camby they reported the incidence of nausea and vomiting to be as high as $40 \% .{ }^{12}$ In our study the incidence of this complication was $1.2 \%$. Major cardiac arrhythmias, myocardial infarction, symptomatic hypoxia which are other complications of hemodialysis were not reported in this study. Symptoms like chest tightness and back ache are also known to be associated with hemodialysis. However no distressing chest pain or backache was noted in our study.

The overall low incidence of complications during hemodialysis seen in our patients may be due to the small sample size. Tolerance on the part of the patients may have contributed to the underreporting of minor complications. The higher mortality in this study group might mean that patients were quite sick and minor complications may have been underreported on part of the care giver. Further large scale studies are needed to address this issue better in the future. More stringent criteria for defining a complication and reporting it may increase the incidence of complications.

\section{CONCLUSIONS}

The present study however outlines the fact that complications do occur during hemodialysis but they are not very common in our patients and many of them are not of a severe nature. So we can conclude that hemodialysis with all its advancements is a safe and tolerable procedure in the management of ESRD patients.

\section{REFERENCES}

1. Levin A, Hemmelgarn B, Culleton B, Tobe S, McFarlane P, Ruzicka M, et al.: Guidelines for the management of chronic kidney disease. CMAJ. 2008;179:1154-62.

2. Warnock Dg. Chronic renal failure .In: Bunnet et al. Cecil textbook of medicine. $20^{\text {th }}$ ed. Philadelphia: WB Saunders; 1996. P. 556-63.

3. Scribner BH, Caner JE, Buri R, Quinton W. The technique of continuous hemodialysis. Trans Am Soc Artif Intern Organs. 1960;6:88-103.

4. Hada R. Kidney transplantation in Nepal. PMJN. 2003;4:51-4.

5. Davenport A. Intradialytic complications during hemodialysis. Hemodialysis international. 2006;10: 162-7.

6. Jindal KK, Manuel A, Goldstein MB. Percentage reduction for blood urea concentration during hemodialysis (PRU). A simple and accurate method to estimate Kt/v urea. Trans. Am. Soc. Artis Int Organ. 1987;33:286-8.
7. Carpenter CB,Lazarus JM. Dialysis and transplant in the treatment of renal failure. In:Harrison's principles of internal medicine. New York: McGraw Hill; 1998. p. 1520-9.

8. Chhetri PK,Manandhar D,Tiwari $\mathrm{R}$ et al. In centre hemodialysis for ESKD at Nepal medical college and Teaching hospital. Nepal Med Coll J. 2009;11(1):61-3.

9. Ahmed A,Khan AR, Mustafa G. The frequencies of complications during dialysis. Pakistan J Med Res. 2002;41:17-23.

10. Collins DM, Lamber MB.Tolerence of hemodialysis. J Am Soc Nephrol. 1993;4(2):148-54.

11. Himmelfarb J. Hemodialysis complications. American Journal of Kidney Diseases. 2005;45(6):1122-31.

12. Salvatored D, Vincenjo C. Hemodialysis and peritoneal dialysis. Med Int. 1995; 9(30):151-4. 